\title{
Intelligent Inventory Control: Is Bootstrapping Worth Implementing?
}

\author{
Tatpong Katanyukul ${ }^{1}$, Edwin K.P. Chong ${ }^{2}$, and William S. Duff ${ }^{3}$ \\ ${ }^{1}$ Embedded System Research Group and Department of Computer Engineering, \\ Faculty of Engineering, Khon Kaen University, Khon Kaen, Thailand \\ tatpong@kku.ac.th \\ ${ }^{2}$ Department of Electrical and Computer Engineering, College of Engineering, \\ Colorado State University, Fort Collins, CO, USA \\ edwin. chong@colostate.edu \\ ${ }^{3}$ Department of Mechanical Engineering, College of Engineering, Colorado State University, \\ Fort Collins, CO, USA \\ bill@engr.colostate.edu
}

\begin{abstract}
The common belief is that using Reinforcement Learning methods (RL) with bootstrapping gives better results than without. However, inclusion of bootstrapping increases the complexity of the RL implementation and requires significant effort. This study investigates whether inclusion of bootstrapping is worth the effort when applying RL to inventory problems. Specifically, we investigate bootstrapping of the temporal difference learning method by using eligibility trace. In addition, we develop a new bootstrapping extension to the Residual Gradient method to supplement our investigation. The results show questionable benefit of bootstrapping when applied to inventory problems. Significance tests could not confirm that bootstrapping had statistically significantly reduced costs of inventory controlled by a RL agent. Our empirical results are based on a variety of problem settings, including demand correlations, demand variances, and cost structures.
\end{abstract}

Keywords: approximate dynamic programming, inventory control, reinforcement learning, bootstrapping, eligibility trace, intelligent agent.

\section{Introduction}

Inventory management is one of the major business activities. A well managed inventory can help a business stay competitive by keeping its cash flow at a controllable level. A stochastic multiperiod inventory problem-one of the most common inventory problems - can be modeled as a Markov Decision Problem (MDP). Approximate Dynamic Programming (ADP) is a method to solve practical Markov decision problems. Most previous studies on ADP applied to inventory management focused on learning scheme, which is also known as Reinforcement Learning (RL). Due to its effectiveness and its link to mammal learning processes, temporal difference learning, or sometimes called "one-step temporal-difference learning", $\mathrm{TD}(0)$, is one of the most widely studied RL methods. 
Eligibility trace has been used to bootstrap the learning process of TD(0). The integration of the eligibility trace into the temporal difference learning leads to the $\operatorname{TD}(\lambda)$ method. The success of $\operatorname{TD}(\lambda)$ in many applications has lead to a general belief (see, e.g., Prestwich et al. (2008)) that using $\operatorname{TD}(\lambda)$ with $\lambda$ between 0 and 1 will give better results than $\operatorname{TD}(0)$. However, the effort required to implement $\operatorname{TD}(\lambda)$ is considerably higher than to implement $\operatorname{TD}(0)$. The value and benefit of using $\operatorname{TD}(\lambda)$ compared to $\mathrm{TD}(0)$ have never been investigated especially for inventory management.

Our study investigates the application of $\operatorname{Sarsa}(\lambda)$, a widely studied implementation of the $\operatorname{TD}(\lambda)$ method. To supplement the investigation, our study develops the Direct Credit Back method (DCB) - the bootstrapped version of the Residual Gradient method (RG). Finally, we evaluate both bootstrapping methods-Sarsa $(\lambda)$ and DCB - and confer to their non-bootstrapped counterparts, Sarsa and RG. The underlying methods are evaluated with various structures of inventory problems. Two other inventory control methods-Look-Ahead and Rollout-are also included in our study.

The findings here provide a practical approach to apply an ADP method for an inventory problem. The results reveal questionable benefits of bootstrapping. The understanding exposed here will help promote efficient inventory management and aid in the transfer of intelligent system research into practice.

\section{Literature Review}

ADP has been introduced recently into inventory management research. Kim et al. (2005), Kim et al. (2008), Kwon et al. (2008), and Jiang and Sheng (2009) implemented $\mathrm{TD}(0)$ with a look-up table as a cost-to-go approximation-a crucial component of most RL methods. A look-up table is simple to implement and works well with $\mathrm{TD}(0)$. However, a look-up table suffers from a scalability issue: its memory requirement grows exponentially as the dimension of the problem space grows.

Some studies, e.g., Van Roy et al. (1997) and Shervais et al. (2003), apply TD(0) with nonlinear function approximation, such as artificial neural network, to mitigate a scalability issue. However, applying $\mathrm{TD}(0)$ with nonlinear function approximation requires a high level of expertise in both application and techniques and it might result in divergence leading to instability of the control. Baird (1995) proposed Residual Gradient method (RG) to be used with nonlinear function approximation. However, $\mathrm{RG}$ is reported to underperform $\mathrm{TD}(0)$ in overall.

In addition to extend RL method with scalable function approximation, bootstrapping is used to speed up the learning process of $\mathrm{TD}(0)$ and leads to a more general method, denoted $\operatorname{TD}(\lambda)$. Successes of applying $\operatorname{TD}(\lambda)$ - the most famous work is Tesauro (1994) - lead to common belief of the benefit of bootstrapping. However, due to complexity of implementation and extra computational costs, there is only work of Prestwich et al. (2008) related to bootstrapping and inventory management. Prestwich et al. (2008) studied the viability of combining $\operatorname{Sarsa}(\lambda)$ and Noisy Genetic Algorithm by using the Cultural Algorithm, introduced by Reynolds (1994). They claimed the viability of their approach for partially observable Markov decision problems. 
Among previous authors applying ADP to inventory problems, many authors have used $\operatorname{TD}(0)$ or methods related to $\operatorname{TD}(0)$, but none ${ }^{1}$ has investigated the effectiveness of bootstrapping. Leng et al. (2009) also mentioned that the mechanism of Eligibility Trace in ADP with function approximation has not been sufficiently investigated.

Inspired by the development of eligibility trace to bootstrap $\mathrm{TD}(0)$, we develop an extension to RG based on bootstrapping, called "Direct Credit Back" (DCB). The new method DCB provides a contribution in its own right as well as supplements the investigation of an effect of bootstrapping in applying RL to inventory management.

\section{Background}

Sarsa. Sarsa, as discussed by Sutton and Barto (1998), uses an approximate stateaction cost $Q\left(s_{t}, a_{t}\right)$, often called the Q-value, to determine an action and updates $Q\left(s_{t}\right.$, $a_{t}$ ) based on $\operatorname{TD}(0)$. The equations for Sarsa are $Q(s, a) \leftarrow Q(s, a)+\beta \psi(s, a)$ where $Q(s, a)$ approximates the state-action cost of state $s$ and action $a ; \beta$ is a Sarsa parameter, called the learning rate; the temporal difference $\psi(s, a)=c(s, a)+\alpha Q\left(s^{\prime}, a^{\prime}\right)-$ $Q(s, a)$ when $c(s, a)$ is a single period cost and action $a_{t}$ is determined by a chosen policy based on state $s_{t}$ and the current Q-value.

Eligibility Trace. $\mathrm{TD}(0)$ uses newly observed information, $c\left(s_{t}, a_{t}\right)$, to update a $\mathrm{Q}-$ value of the most recent state-action. To utilize trajectory information, Eligibility Trace (ET) is developed. $\mathrm{ET}^{2}$ updates its values, such that when $(s, a)$ is visited, eligibility variable $e(s, a) \leftarrow 1, e(s, \hat{a}) \leftarrow 0$ for each action $\hat{a} \neq a$, and $e(\hat{s}, b) \leftarrow \alpha \lambda e(\hat{s}$, $b$ ) for each state $\hat{s} \neq s$ and each action $b \in A(\hat{s})$ where $\alpha$ is a discount factor; $\lambda$ is an eligibility factor; and $\mathrm{A}(\hat{s})$ is a feasible action set for the given state $\hat{s}$. Sarsa $(\lambda)$ is an implementation of $\operatorname{TD}(\lambda)$, whose the update equation is $Q(s, a) \leftarrow Q(s, a)+\beta \psi(s, a)$ $e(s, a)$ for all $s$ and $a$.

Residual Gradient Method. Sarsa was originally designed to be implemented using a look-up table and later was extended for use with an approximation function. Although there are some success, associated risk of instability is commonly known, see Baird (1995), Barreto and Anderson (2008), and Maei et al. (2009). The Residual Gradient method (RG), introduced by Baird (1995), is designed to be used with various approximation functions, including ones belonging to a non-linear family. RG is developed to minimize the approximation error $^{3} \xi(\theta)=\sum_{t=1, \ldots, T} \xi_{t}(\theta)$ where $\xi(\theta)$ is the total approximation error and $\xi_{t}(\theta)=\left\{C\left(s_{t}\right)-Q\left(s_{t} \mid \theta\right)\right\}^{2} / 2$ is the approximation error of period $t$. Therefore, the parameter values can be determined by gradient descend method: $\theta \leftarrow \theta-\beta \cdot\left\{C\left(s_{t}\right)-Q\left(s_{t} \mid \theta\right)\right\} \nabla_{\theta}\left\{C\left(s_{t}\right)-Q\left(s_{t} \mid \theta\right)\right\}$. In the development of Sarsa, partial differentiation is applied, then the real state cost $C\left(s_{t}\right)$ is approximated by $c\left(s_{t}\right)+\alpha Q\left(s_{t+1}\right)$. In development of $\mathrm{RG}$, the approximation is

1 Prestwich et al. (2008) used Sarsa( $\lambda$ ) only as a competing method with hill-climbing method to determine the parameter values of $\operatorname{Sarsa}(\lambda)$, but have not investigated the effectiveness of bootstrapping.

2 ET presented here is based on Singh and Sutton (1996)'s replacing trace.

3 To be concise, the content here is presented based on state costs. The development based on state-action costs can be conducted in a similar manner. 
applied before differentiation. The RG update equation is $\theta \leftarrow \theta+\beta \cdot \psi\left(s_{t}\right) \cdot\left\{\alpha \nabla_{\theta}\right.$ $\left.Q\left(s_{t+1} \mid \theta\right)-\nabla_{\theta} Q\left(s_{t} \mid \theta\right)\right\}$ where $\psi\left(s_{t}\right)=c\left(s_{t}\right)+\alpha Q\left(s_{t+1} \mid \theta\right)-Q\left(s_{t} \mid \theta\right)$.

\section{Direct Credit Back}

Baird (1995) claims that RG always converges. However, it has been criticized for delivering an inferior solution compared to TD(0) by Maei et al. (2009). To improve the solution obtained from RG, we develop a Direct Credit Back (DCB) method based on bootstrapping. The DCB method uses the newly observed datum $c\left(s_{t}\right)$ to update the approximate costs of the most recent state as well as other prior states.

The temporal difference error of the approximate cost of state $s_{t}$ is

$$
\psi\left(\mathrm{s}_{\mathrm{t}}\right)=\mathrm{c}\left(\mathrm{s}_{\mathrm{t}}\right)+\alpha \mathrm{Q}\left(\mathrm{s}_{\mathrm{t}+1} \mid \theta\right)-\mathrm{Q}\left(\mathrm{s}_{\mathrm{t}} \mid \theta\right) .
$$

After the period cost $c\left(s_{t}\right)$ is observed, $Q\left(s_{t} \mid \theta\right)$ can be approximated by $c\left(s_{t}\right)+\alpha$ $Q\left(s_{t+1} \mid \theta\right)$. Therefore, the temporal difference error of the approximate cost of state $s_{t-1}$ after $c\left(s_{t}\right)$ is observed is defined as

$$
\psi\left(\mathrm{s}_{\mathrm{t}-1} \mathrm{lc}\left(\mathrm{s}_{\mathrm{t}}\right)\right)=\mathrm{c}\left(\mathrm{s}_{\mathrm{t}-1}\right)+\alpha\left\{\mathrm{c}\left(\mathrm{s}_{\mathrm{t}}\right)+\alpha \mathrm{Q}\left(\mathrm{s}_{\mathrm{t}+1} \mid \theta\right)\right\}-\mathrm{Q}\left(\mathrm{s}_{\mathrm{t}-1} \mid \theta\right) .
$$

Equation (2) can be rearranged as shown in Equation (3),

$$
\psi\left(\mathrm{s}_{\mathrm{t}-1} \mid \mathrm{c}\left(\mathrm{s}_{\mathrm{t}}\right)\right)=\mathrm{c}\left(\mathrm{s}_{\mathrm{t}-1}\right)+\alpha\left\{\psi\left(\mathrm{s}_{\mathrm{t}} \mid \mathrm{c}\left(\mathrm{s}_{\mathrm{t}}\right)\right)+\mathrm{Q}\left(\mathrm{s}_{\mathrm{t}} \mid \theta\right)\right\}-\mathrm{Q}\left(\mathrm{s}_{\mathrm{t}-1} \mid \theta\right)
$$

where $\psi\left(s_{t} \mid c\left(s_{t}\right)\right)=\psi\left(s_{t}\right)$.

The update equations for other prior states can be obtained in a similar manner. After $c\left(s_{t}\right)$ is observed, the temporal difference error of approximate cost of state $s_{t-i}$, for $i=1,2, \ldots, t-1$, is shown in Equation (4):

$$
\psi\left(\mathrm{s}_{\mathrm{t}-\mathrm{i}} \mathrm{l}\left(\mathrm{s}_{\mathrm{t}}\right)\right)=\mathrm{c}\left(\mathrm{s}_{\mathrm{t}-\mathrm{i}}\right)+\alpha\left\{\psi\left(\mathrm{s}_{\mathrm{t}-\mathrm{i}+1} \mathrm{l}\left(\mathrm{s}_{\mathrm{t}}\right)\right)+\mathrm{Q}\left(\mathrm{s}_{\mathrm{t}-\mathrm{i}+1} \mid \theta\right)\right\}-\mathrm{Q}\left(\mathrm{s}_{\mathrm{t}-\mathrm{i}} \mid \theta\right) .
$$

Minimizing the squared temporal difference errors of all prior states is equivalent to minimizing $\sum_{i=0, \ldots, t-l} \psi^{2}\left(s_{t-i} \mid c\left(s_{t}\right)\right)$. The DCB update equation can be obtained by the gradient descent method. The update can be truncated to only a specific number of prior approximate state costs,

$$
\theta \leftarrow \theta-\beta \sum_{\mathrm{i}=0, \ldots, \min \{\mathrm{t}-1, \mathrm{~N}\}} \psi\left(\mathrm{s}_{\mathrm{t}-\mathrm{i}} \mathrm{lc}\left(\mathrm{s}_{\mathrm{t}}\right)\right) \cdot\left\{\alpha^{\mathrm{i}+1} \cdot \nabla_{\theta} \mathrm{Q}\left(\mathrm{s}_{\mathrm{t}+1} \mid \theta\right)-\nabla_{\theta} \mathrm{Q}\left(\mathrm{s}_{\mathrm{t}-\mathrm{i}} \mid \theta\right)\right\}
$$

where $N \in\{0,1,2, \ldots\}$ is the number of periods crediting back. The update equation for approximate state-action costs can be obtained in a similar manner. When the parameter $N=0$, the DCB method reduces to RG.

\section{Experiments}

Our study uses computer simulations to conduct numerical experiments. The inventory problem investigated here is a periodic review single-echelon problem with nonzero leadtime and a setup cost. 
The demand is modeled as $\operatorname{AR} 1 / \operatorname{GARCH}(1,1)$. Therefore, a state is composed of the previous demand $D_{t-1}$, the previous demand error $\varepsilon_{t-l}$, the variance of the previous demand error $\sigma_{t-l}^{2}$, the on-site inventory $x_{t}$, and the in-transit inventory $B^{(t)}$ whose length depends on a leadtime $L$. The state space of this problem is $\left\{0, I^{+}\right\} \times R \times R \times I$ $\times\left\{0, I^{+}\right\}^{L}$ for $D_{t-l}, \varepsilon_{t-l}, \sigma_{t-l}^{2}, x_{t}$, and $B^{(t)}$, respectively. An action $u_{t} \in\left\{0, I^{+}\right\}$is a replenishment order. State transitions are specified by (1) $D_{t}=a_{0}+a_{1} D_{t-1}+\varepsilon_{t}$, (2) $\varepsilon_{t}=e_{t}$ $\sigma_{t}$, (3) $\sigma_{t}^{2}=v_{0}+v_{l} \varepsilon_{t-1}{ }^{2}+v_{2} \sigma_{t-1}{ }^{2}$, (4) $x_{t+1}=x_{t}+B^{(t)}{ }_{1}-D_{t}$, and (5) $B^{(t+1)}=\left[B^{(t+1)}{ }_{1} \ldots\right.$

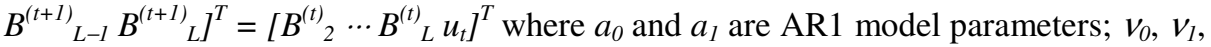
and $v_{2}$ are $\operatorname{GARCH}(1,1)$ parameters; $e_{t}$ is white noise distributed according to $N(0$, 1 ); and $u_{t}$ is the replenishment order.

The period cost consists of the replenishment cost and the inventory handling cost, $c_{t+1}=K_{t} \delta\left(u_{t}\right)+g_{t} u_{t}+h_{t}\left(x_{t+1}\right)^{+}+b_{t}\left(-x_{t+1}\right)^{+}$where $c_{t+1}$ is the period cost, whose value will be known at time $t+1$ (the end of period t); $K_{t}$ is the setup cost; $g_{t}$ is the unit replenishment cost; $h_{t}$ is the unit holding cost; $b_{t}$ is the unit backlogging cost; $\delta(\cdot)$ is the step function; $(\cdot)^{+}$is the positive function defined by $(a)^{+}=a \delta(a)$; and other variables are as mentioned earlier.

Our study investigated 13 different inventory problem structures (as shown in Table 1) with other parameters held fixed: AR1's $a_{0}=2, \operatorname{GARCH}(1,1)$ 's $v_{l}=0.1$ and $v_{2}=0.8$, a discount factor $\alpha=1$, a leadtime $L=1$, and the unit replenishment cost $g=$ $\$ 100 /$ unit. Each experiment is initialized at $D_{0}=50, \varepsilon_{0}=10, \sigma_{0}^{2}=400, x_{1}=10$, and $B^{(1)}=0$. Each method's performance is evaluated based on its aggregate cost of Periods 13-60. Using an aggregate cost allows ADP methods to have initial learning

Table 1. A summary of demand and cost variables investigated

\begin{tabular}{|l|c|c|c|c|c|}
\hline & \multicolumn{2}{|c|}{ demand variables } & \multicolumn{3}{c|}{ cost variables } \\
\hline & $\begin{array}{c}\text { correlation } \\
\left(\mathrm{a}_{1}\right)\end{array}$ & $\begin{array}{c}\text { noise variance offset } \\
\left(\mathrm{v}_{0}\right)\end{array}$ & $\begin{array}{c}\text { set up cost } \\
(\mathrm{K}: \$ / \text { transaction })\end{array}$ & $\begin{array}{c}\text { penalty cost } \\
(\mathrm{b}: \$ \text { /item })\end{array}$ & $\begin{array}{c}\text { holding cost } \\
(\mathrm{h}: \$ \text { /item })\end{array}$ \\
\hline P1 & 0.8 & 70 & 100 & 200 & 1 \\
\hline P2 & 0 & 70 & 100 & 200 & 1 \\
\hline P3 & -0.8 & 70 & 100 & 200 & 1 \\
\hline P4 & 0.8 & 40 & 100 & 200 & 1 \\
\hline P5 & 0.8 & 10 & 100 & 200 & 1 \\
\hline P6 & 0.8 & 70 & 150 & 100 & 1 \\
\hline P7 & 0.8 & 70 & 100 & 100 & 1 \\
\hline P8 & 0.8 & 70 & 50 & 100 & 1 \\
\hline P9 & 0.8 & 70 & 0 & 100 & 1 \\
\hline P10 & 0.8 & 70 & 0 & 200 & 1 \\
\hline P11 & 0.8 & 70 & 0 & 50 & 1 \\
\hline P12 & 0.8 & 70 & 0 & 50 & 10 \\
\hline P13 & 0.8 & 70 & 0 & 50 & 25 \\
\hline
\end{tabular}


periods and thus provides better performance evaluation of ADP methods. The experiments run each inventory controller for 50 replications of each of 60 time-unit-indeterminate periods.

Implementation. Sarsa, $\operatorname{Sarsa}(\lambda), \mathrm{RG}$, and DCB methods are used with the Radial Basis Function (RBF) as the approximation function. RBF has three sets of parameters: centers, scales, and weights. RBF centers and scales are set up as Katanyukul et al. (2011). RBF weights are adjustable parameters whose values are determined by the method under investigated. Variables $\varepsilon_{\mathrm{t}-1}$ and $\sigma_{\mathrm{t}-1}{ }^{2}$ are excluded from state parameters for reasons discussed in Katanyukul et al. (2011).

\section{Experimental Results}

We measure performance of each inventory control by aggregate costs. Figures 1 and 2 show averages and confidence intervals of aggregate costs obtained from different methods under problem scenarios P1-P13 (Table 1). A '*' marks an average aggregate cost and line beside it represents a $90 \%$ confidence interval, based on t-test statistics. On the y-axis, labels 'H', 'S', 'SL', 'RG', and 'DCB', and 'Roll' indicate results obtained from the 12-period Look-Ahead, Sarsa, Sarsa $(\lambda), R G$, and the DCB method, respectively ${ }^{4}$. The Roll-out method was used with perfect system information: Rollout simulation parameter values match those of the actual problem.
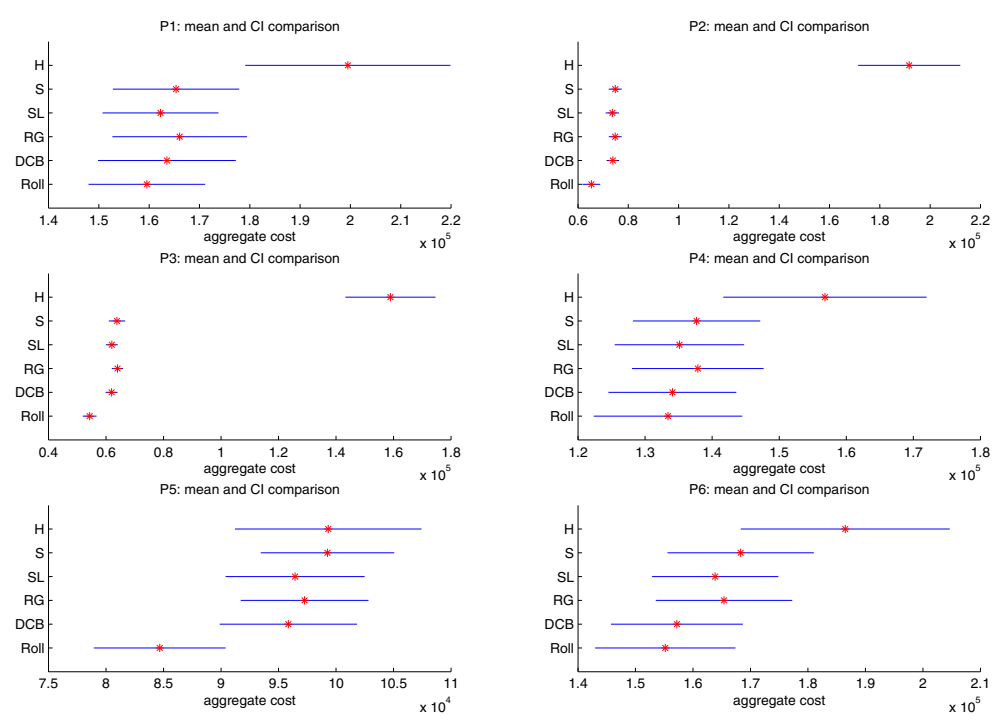

Fig. 1. Means and Confidence Intervals of aggregate costs

\footnotetext{
4 Results of each method are presented with the best performing set of parameter values.
} 

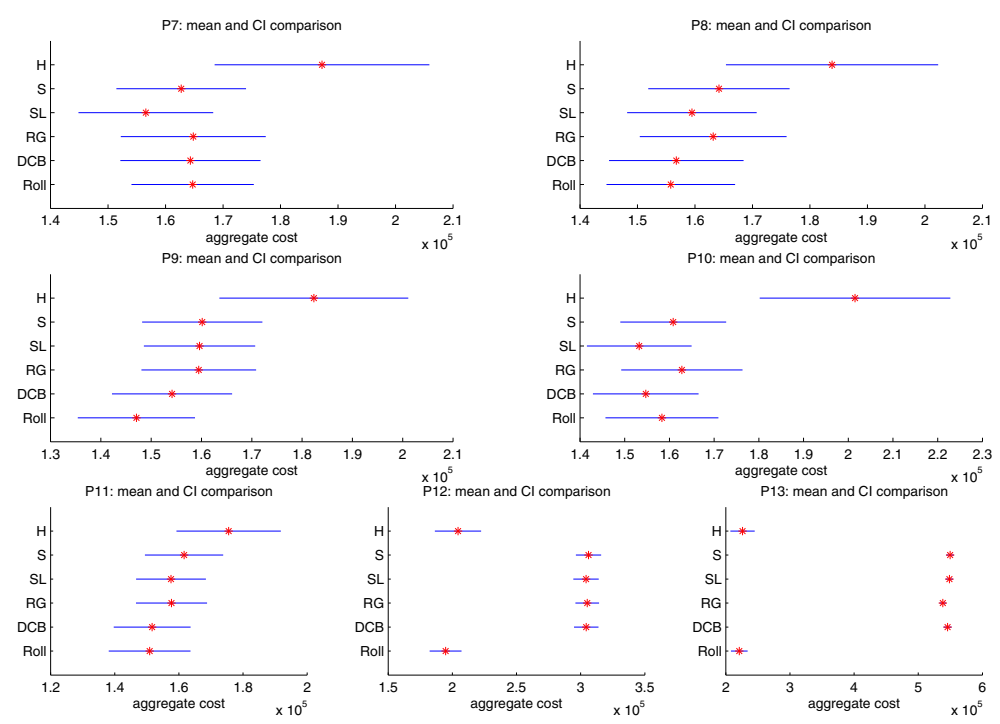

Fig. 2. Means and Confidence Intervals of aggregate costs

\section{Discussion and Conclusions}

The average costs obtained from RL methods with bootstrapping are lower than without in most scenarios, but P13 that the average cost obtained from DCB is higher than one from RG method. Figures 3 and 4 illustrate the effect of bootstrapping. The $\mathrm{y}$-axes show the percentage relative cost difference of method A to method B, denoted "\% rel. cost diff.", which is $\left(\operatorname{cost}_{A} / \operatorname{cost}_{B}-1\right) \times 100 \%$. The x-axes show scenario parameters as indicated: demand correlations $\left(a_{l}\right)$, variances $\left(v_{0}\right)$, set up per unit cost $(K / g)$, penalty cost per unit cost $(b / g)$, and holding cost per penalty cost $(h / b)$. The plots in the first columns show \% rel. cost diff. of each RL method, as indicated by the legends, to the 12-period Look-Ahead method (H12). Similarly, the plots in the middle and last columns show \% rel. cost diff. to Sarsa (S) and the Residual Gradient method (RG), respectively.

Based on percentages of relative cost difference, bootstrapping reduces average cost up to about $5 \%$, regardless of the RL method. It should be noted that scenario P13 that DCB has higher average cost than RG is when the holding cost ratio $(h / b)$ is relatively high. When the holding cost is relatively high, not only that DCB underperforms RG method, all methods-Sarsa, $\operatorname{Sarsa}(\lambda), \mathrm{RG}$, and DCB methodsunderperform the 12-period Look-Ahead method (bottom left plot of Figure 4). This implies that RL method does not work well under high $h / b$ ratio. High $h / b$ ratios make inventory decisions more critical—stocking more inventory has more negative effect. This may cause slower convergence of all RL methods. The study of suitable ADP methods for critical decision problems deserves further investigation. 

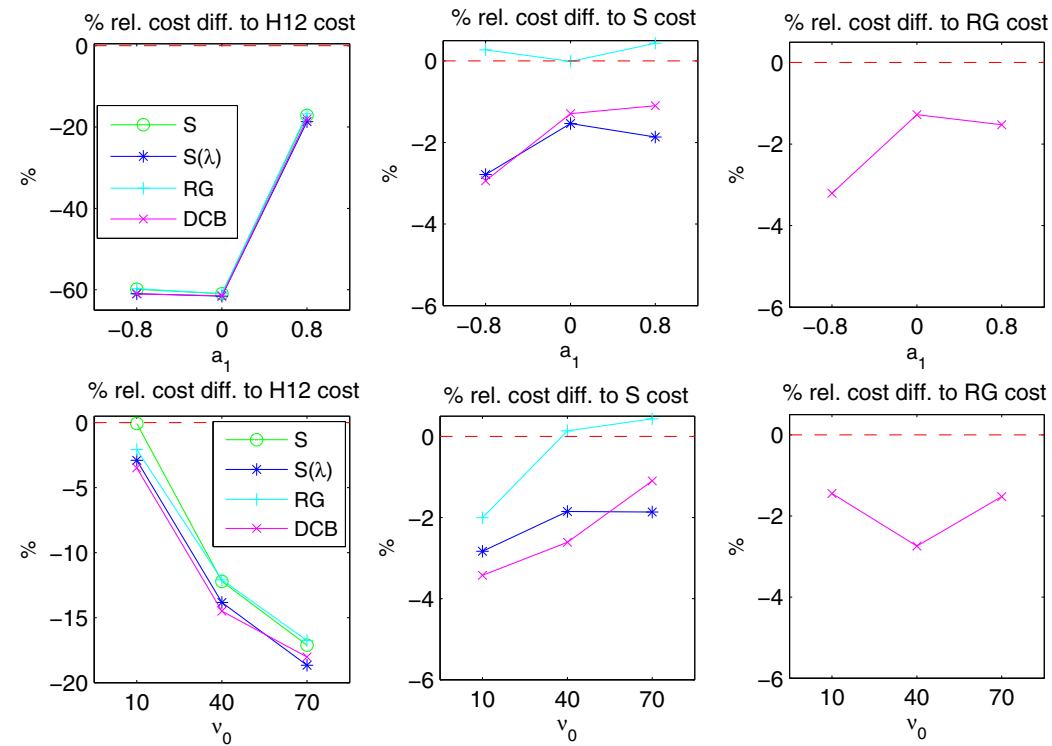

Fig. 3. Relative cost differences of ADP methods on different demand correlations (upper row) and variances (lower row)
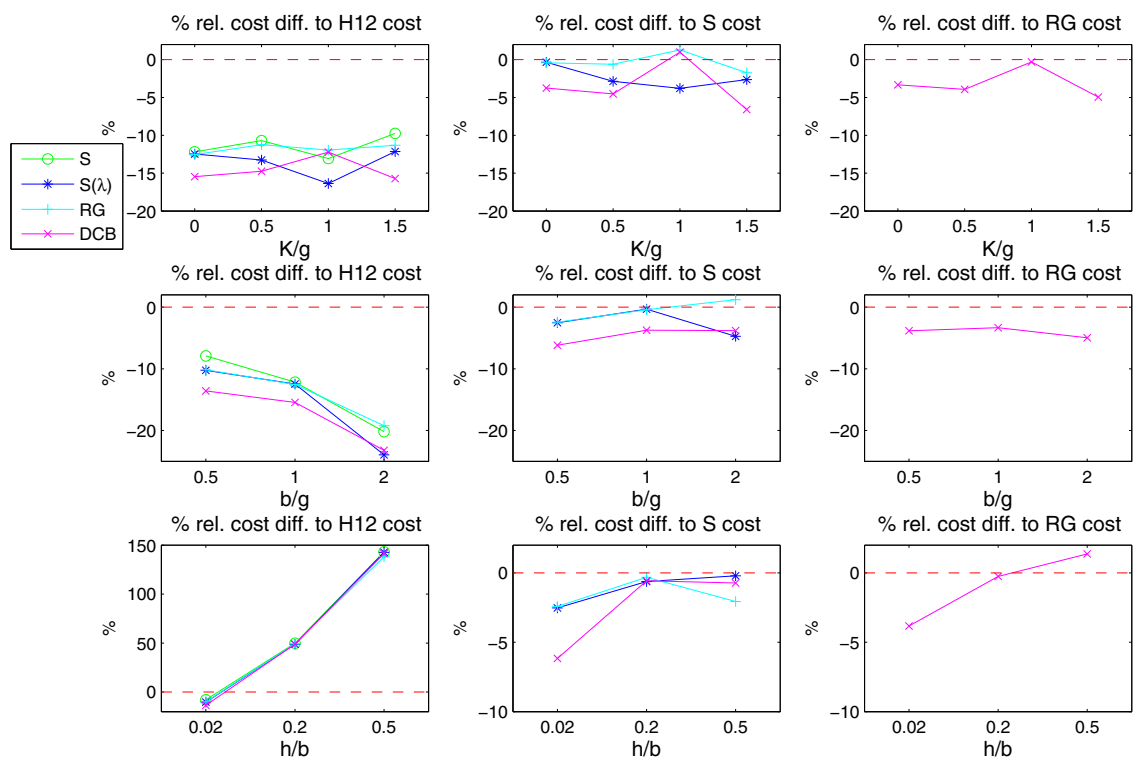

Fig. 4. Relative cost differences of ADP methods on different set up costs (top row), penalty costs (middle row) and holding costs (bottom row) 
Although the reduction of average aggregate costs by using bootstrapping seems apparent in most cases, the reduction is still within ranges of variation (at 95\% confidence level). Therefore, statistically we cannot rule out the possibilities of the variation of results due to stochastic variations in the problems. We have run significance tests to double-check, but the test results could not confirm the cost reduction of bootstrapping at $95 \%$ confidence level. Table 2 shows p-values of the significance tests. The higher p-value, i.e., closer to 1 , implies that evidence against the null hypothesis is insufficient.

To conclude the point, bootstrapping has shown to be able to reduce average cost up to $5 \%$ with possibility that this reduction may be due to variation of the underlying process. Therefore, taking into consideration the additional implementation effort and computation cost required by bootstrapping, bootstrapping in the forms investigated in this study are not recommended for inventory control applications.

It should be noted that our conclusion is drawn based on our experimental results. Investigation of different form of bootstrapping or different problem structure, such as a problem with long leadtime, may reveal a situation where bootstrapping can work more effectively.

Table 2. Significance test results

\begin{tabular}{|l|c|c|c|c|c|c|c|}
\hline \multicolumn{7}{|c|}{ p-values obtained from t-tests } \\
\hline Scenario & $\mathrm{P} 1$ & $\mathrm{P} 2$ & $\mathrm{P} 3$ & $\mathrm{P} 4$ & $\mathrm{P} 5$ & $\mathrm{P} 6$ & $\mathrm{P} 7$ \\
\hline $\mathrm{H}_{\mathrm{a}}: \operatorname{cost}_{\mathrm{S}} \neq \operatorname{cost}_{\mathrm{S}(\lambda)}$ & 0.76 & 0.60 & 0.39 & 0.75 & 0.57 & 0.66 & $(0.45)$ \\
\hline $\mathrm{H}_{\mathrm{a}}: \operatorname{cost}_{\mathrm{RG}} \neq \operatorname{cost}_{\mathrm{DCB}}$ & $(0.86)$ & 0.65 & 0.23 & 0.64 & 0.77 & $(0.39)$ & $(0.97)$ \\
\hline Scenario & $\mathrm{P} 8$ & $\mathrm{P} 9$ & $\mathrm{P} 10$ & $\mathrm{P} 11$ & $\mathrm{P} 12$ & $\mathrm{P} 13$ & \\
\hline $\mathrm{H}_{\mathrm{a}}: \operatorname{cost}_{\mathrm{S}} \neq \operatorname{cost}_{\mathrm{S}(\lambda)}$ & 0.63 & 0.96 & 0.44 & $(0.66)$ & 0.82 & $(0.69)$ & \\
\hline $\mathrm{H}_{\mathrm{a}}: \operatorname{cost}_{\mathrm{RG}} \neq \operatorname{cost}_{\mathrm{DCB}}$ & $(0.71)$ & $(0.52)$ & $(0.58)$ & $(0.43)$ & 0.92 & 0.12 & \\
\hline
\end{tabular}

Remark: p-values in parentheses represent p-values obtained from Wilcoxon ranksum tests. Wilcoxon ranksum test is used when assumption of normality is doubted. The normal assumption is tested by Lilliefor test at $5 \%$ significance level.

\section{References}

1. Baird, L.: Residual Algorithms: Reinforcement Learning with Function Approximation. In: Proceedings of the 12th International Conference on Machine Learning, pp. 30-37. Morgan Kaufmann (1995)

2. Barreto, A.M.S., Anderson, C.W.: Restricted gradient-descent algorithm for value-function approximation in reinforcement learning. Artificial Intelligence 172(4-5), 454-482 (2008)

3. Jiang, C., Sheng, Z.: Case-based reinforcement learning for dynamic inventory control in a multi-agent supply chain system. Expert Systems with Applications 36(3), 6520-6526 (2009)

4. Katanyukul, T., Duff, W.S., Chong, E.K.P.: Approximate dynamic programming for an inventory problem: Empirical comparison. Computers \& Industrial Engineering 60(4), 719-743 (2011) 
5. Kim, C.O., Jun, J., Baek, J.K., Smith, R.L., Kim, Y.D.: Adaptive inventory control models for supply chain management. International Journal of Advanced Manufacturing Technology 26(9-10), 1184-1192 (2005)

6. Kim, C.O., Kwon, I.H., Baek, J.G.: Asynchronous action-reward learning for nonstationary serial supply chain inventory control. Applied Intelligence 28(1), 1-16 (2008)

7. Kwon, I.H., Kim, C.O., Jun, J., Lee, J.H.: Case-based myopic reinforcement learning for satisfying target service level in supply chain. Expert Systems with Applications 35(1-2), 389-397 (2008)

8. Leng, J., Jain, L., Fyfe, C.: Experimental analysis of eligibility traces strategies in temporal difference learning. International Journal of Knowledge Engineering and Soft Data Paradigms 1(1), 26-39 (2009)

9. Maei, H.R., Szepesvari, C., Bhatnagar, S., Precup, D., Silver, D., Sutton, R.S.: Convergent Temporal-Difference Learning with Arbitrary Smooth Function Approximation. In: Advances in Neural Information Processing Systems. MIT Press, Vancouver (2009)

10. Prestwich, S.D., Tarim, S.A., Rossi, R., Hnich, B.: A Cultural Algorithm for POMDPs from Stochastic Inventory Control. In: Blesa, M.J., Blum, C., Cotta, C., Fernández, A.J., Gallardo, J.E., Roli, A., Sampels, M. (eds.) HM 2008. LNCS, vol. 5296, pp. 16-28. Springer, Heidelberg (2008)

11. Reynolds, R.G.: An Introduction to Cultural Algorithms. In: Proceedings of the 3rd Annual Conference on Evolutionary Programming, pp. 131-139. World Scientific Publishing (1994)

12. Shervais, S., Shannon, T.T., Lendaris, G.G.: Intelligent Supply Chain Management Using Adaptive Critic Learning. IEEE Transactions on Systems, Man, and Cybernetics-Part A: Systems and Humans 33(2), 235-244 (2003)

13. Singh, S.P., Sutton, R.S.: Reinforcement Learning with Replacing Eligibility Traces. Machine Learning 22(1-3), 123-158 (1996)

14. Sutton, R.S., Barto, A.G.: Reinforcement Learning. MIT Press (1998)

15. Tesauro, G.J.: TD-Gammon, a self-teaching backgammon program, achieves master level play. Neural Computation 6(2), 215-219 (1994)

16. Van Roy, B., Bertsekas, D.P., Lee, Y., Tsitsiklis, J.N.: A Neuro-Dynamic Programming Approach to Retailer Inventory Management. In: Proceedings of the IEEE Conference on Decision and Control (1997) 